\title{
Clinical applications of DNA methylation biomarkers in colorectal cancer
}

"...recent DNA methylation biomarker studies show promising results that have the potential to impact the way colorectal cancer patients will be treated in the future."

\section{KEYWORDS: biomarkers = colorectal cancer $\approx$ DNA methylation - epigenetics - epigenomics}

Colorectal cancer (CRC) is a common disease that results in significant morbidity and mortality worldwide. CRC is the second leading cause of cancer mortality worldwide, and accounts for over 600,000 deaths annually [1]. Biomarkers are molecules or substances found in blood, other bodily fluids or tissues that reflect a particular biological or pathological state. Blood- or stool-based biomarkers have the potential to revolutionize CRC diagnostics by providing noninvasive, patient-friendly and cost-effective early detection of CRC. Cancer biomarkers may have broader applications, including disease risk analysis in otherwise healthy people, molecular classification, therapy response prediction, disease prognostics and recurrence monitoring in patients who have been treated for cancer. In recent years, abnormal patterns of DNA methylation have emerged as candidate cancer biomarkers [2]. Here, we will summarize recent advances in the use of DNA methylation biomarkers in CRC clinical applications.

\section{"The field of DNA methylation biomarker research is evolving rapidly and organized efforts are being made to structure biomarker research and development."}

Germline mutations in hereditary CRC syndromes have proven valuable in identifying individuals at high risk of developing CRC, enabling personalized surveillance and early intervention. These classic high-risk alleles are mostly caused by loss-of-function coding-region mutations. By contrast, germline polymorphisms in regulatory regions of the $M L H 1$ gene have been found to result in a higher likelihood of epigenetic silencing from the affected $M L H 1$ allele, thereby increasing the risk of developing mismatch-repair-deficient CRC [3,4]. In rare cases, the effect of the polymorphism is strong enough to cause constitutional silencing of the MLH1 allele [3], which would be detectable in a blood-based MLH1 DNA methylation assay. Another example of a systemic epigenetic alteration is loss of imprinting of the IGF2 gene, which results in the activation of the normally silent allele of the IGF2 gene, an epigenetic phenomenon associated with various types of cancer, including CRC. Systemic loss of imprinting can be determined in peripheral blood and may be associated with an increased risk of developing CRC [5].

Epigenetic biomarkers have also been explored as cost-effective and noninvasive alternatives to colonoscopy for the early detection of CRC. An ideal early detection DNA methylation biomarker would be present in the targeted malignancy at high frequency and detectable in bodily fluids or secretions despite the presence of background DNA from normal tissues. The most recent DNA methylation biomarker studies show promising results that have the potential to impact the way CRC patients will be treated in the future. The field of DNA methylation biomarker research is evolving rapidly and organized efforts are being made to structure biomarker research and development [6]. In the past, however, many studies have been performed without employing clear rules of evidence, using small sample sizes and relying on candidate gene approaches and nonquantitative detection methods. It has long been known that aberrant patterns of DNA methylation from CRC cells can be detected in tumor-derived cell-free DNA (cfDNA) found in plasma, serum or feces of cancer patients [7]. It is thought that cfDNA in cancer patients is a result of direct shedding from the primary

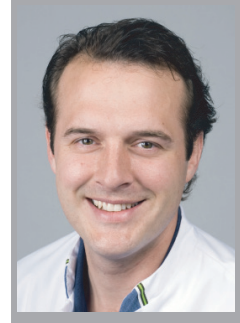

Christopher PE Lange

Leiden University Medical Center, Department of Surgery, Albinusdreef 2, 2333 ZA Leiden, The Netherlands

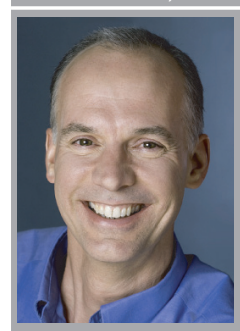

Peter W Laird

Author for correspondence: Department of Surgery, Biochemistry

\& Molecular Biology, Keck School of Medicine of University of Southern California, Norris Comprehensive Cancer Center, 1450 Biggy Street, Room G511B, Los Angeles. CA 90089-9601, USA Tel.: +13234427890 Fax: +13234427880 plaird@usc.edu 
tumor through apoptosis, necrosis or secretion, or potentially originates from free circulating tumor cells or metastatic cell deposits [7]. Studies have reported the existence of specific hypermethylated genes in blood and stool of patients with CRC and their associations with clinical disease and prognosis [8-10]. It has not yet been determined whether the use of a bloodbased test or a stool-based test for early CRC detection is more efficient and more accepted by the public. DNA methylation biomarkers for early detection of CRC have been studied in both blood and feces. A blood-based test has the advantage that it can be used for primary detection, as well as postoperative follow-up for local and distant recurrences. In addition, the processing of a relatively small amount of blood may be preferred in laboratories above handling bulky feces. However, stool-based biomarkers may have some advantages over blood-based tests. Because colorectal adenomas and early CRCs are situated in the inner lining of the colorectum and are in direct contact with feces, tumor secretions and shed cells mix directly with colorectal content. Although it is difficult to estimate whether the half-life of tumor DNA is longer or shorter in feces than in blood, it seems conceivable that the concentration of tumor-derived DNA is higher in the intestine than in the bloodstream. Indeed, reported sensitivities for blood-based CRC DNA methylation biomarkers range up to $90 \%$ with a specificity of $88 \%$ [11]. In stool, sensitivities of $95 \%$ with specificities of $94 \%$ have been published [12]. A recent study by Ahlquist et al. reported a DNA stool test that detects methylated $B M P 3$, NDRG4, vimentin and TFPI2, mutant KRAS, the $A C T B$ gene and the quantity of hemoglobin [13]. This assay detected adenomas larger then $2 \mathrm{~cm}$ with a sensitivity of $82 \%$ and CRC with a sensitivity of $91 \%$ at a specificity of $93 \%$.

\section{"Epigenetic biomarkers have also been explored as cost-effective and noninvasive alternatives to colonoscopy for the early detection of colorectal cancer."}

Little is known about the exact mechanism of tumor cfDNA derivation and spread and its relationship to biomarker detection. Factors that might influence diagnostic performance of a CRC DNA methylation biomarker include tumor, host and test characteristics. It is likely that tumor biology affects detectability. For instance, $\mathrm{CpG}$ island methylator phenotype (CIMP) tumors harbor high frequencies of
DNA methylation and could therefore be more amenable to detection using DNA methylation biomarkers compared with tumors with lower frequencies of aberrant DNA methylation (as is the case for rectal tumors). Furthermore, the total amount of DNA shed by the tumor is likely to influence test performance. In general, biomarker studies show that stage IV CRC tumors, which usually have a higher total tumor burden, are detected more frequently than stage I CRC tumors or adenomas. Although it is known that cfDNA concentrations can vary significantly between individuals, the exact reason why is not clear. Besides individual variances in tumor biology, cfDNA spread and clearance by the liver and kidneys, as well as the wide-ranging diversity of the way samples are treated in different laboratories, may contribute to this variation. With the very low concentrations of tumor-derived DNA in the bloodstream, the volume of blood used in the analysis will affect assay sensitivity. Indeed, most DNA methylation-based detection assays use volumes in the $\mathrm{ml}$ range, rather than the $\mu$ range [2]. For example, the recently commercialized DNA methylation marker SEPT9 is assayed in $4 \mathrm{ml}$ of plasma and yields a sensitivity of $90 \%$ for the detection of CRC at a specificity of $88 \%$ [11,14]. Methylated SFRP2, on the other hand, was reported to detect $67 \%$ of CRCs at a specificity of $94 \%$ using only $200 \mu \mathrm{l}$ of serum [15]. In a recently published study we demonstrated that methylated THBD detects $74 \%$ of stage I/II CRCs at a specificity of $80 \%$ using $1 \mathrm{ml}$ of serum [16]. It is difficult to compare these markers without considering the large difference in volume. It is possible that SFRP2 or THBD would outperform SEPT9 at equal volumes. Future studies will have to point out if increasing test volume also increases sensitivity without loss of specificity.

Molecular classification of CRC is possible using various molecular traits identified in CRC tumor samples. Some commonly used classifications are microsatellite instability (MSI), mutations of the TP53, KRAS and $B R A F$ genes. Although chromosomal instability status also reflects a molecular characteristic and is associated with worse prognosis in CRC, it is usually not used as a molecular classifier owing to its heterogeneity and poorly defined characterization. CIMP is seen in a subset of CRC tumors with unusually frequent DNA hypermethylation of a defined set of loci, and was first described in colon cancers more then a decade ago [17]. Although the exact 
molecular mechanisms and causes of CIMP are unknown, this phenomenon in CRC is associated with a specific phenotype (older age, female sex, family history of CRC, proximal location in the colon, mucinous cell differentiation, specific precursor lesions and smoking) and is tightly associated with sporadic MSI, $B R A F$ and KRAS mutations and MLH1 promotor methylation $[18,19]$. CIMP is a CRC classifier, but also has prognostic meaning, and has been studied as a predictive marker in CRC. A prognostic biomarker gives information on tumor biology; a predictive marker tells us how a specific tumor responds to drug treatment. Although studies have suggested that MSI as well as CIMP have a disadvantageous effect on adjuvant fluorouracil (5-FU)-based treatment in CRC patients (predictive), in general $\mathrm{MSI}^{+} \mathrm{CIMP}^{+}$status is associated with better clinical outcome (prognostic) [20]. It is difficult to study the predictive value of MSI or CIMP on 5-FU drug response due to the high degree of overlap between CIMP and MSI and the fact that 5-FU is the most widely used chemotherapy, administered to almost all eligible CRC patients.[21,22]. Recently, Ebert et al. published a specific association of a particular promotor methylation status with therapy response. Hypermethylation of TFAP2E in primary as well as metastatic CRCs was shown to be associated with decreased expression of this gene and nonresponsiveness to 5-FU. The fact that hypermethylation of TFAP2E was observed in $51 \%$ of the patients in this cohort indicates the possible impact this knowledge could have on future CRC treatment. In addition, if this gene was not methylated, it resulted in a sixtimes higher response rate then the total CRC population [23].

The clinical application of DNA methylation biomarkers in CRC is rapidly developing into a competitive scientific field and has been noticed by the commercial industry as a marketable product. We expect DNA methylation biomarkers to take a definitive place in CRC diagnostics in the near future.

\section{Financial \& competing interests disclosure}

PW Laird is inventor of the MethyLight technology, which has been licensed exclusively to Epigenomics $A G$, for use by Epigenomics in colorectal cancer DNA methylation biomarker assays, including SEPT9. PW Laird receives royalties in this context, but he is neither a shareholder nor consultant for Epigenomics $A G$, and receives no other financial support from Epigenomics $A G$. Epigenomics $A G$ did not contribute to this manuscript in any way. The authors have no other relevant affiliations or financial involvement with any organization or entity with a financial interest in or financial conflict with the subject matter or materials discussed in the manuscript apart from those disclosed.

No writing assistance was utilized in the production of this manuscript.

\section{References}

1 Jemal A, Bray F, Center MM, Ferlay J, Ward E, Forman D. Global cancer statistics. $C A$ Cancer J. Clin. 61(2), 69-90 (2011).

2 Draht MX, Riedl RR, Niessen $\mathrm{H}$ et al. Promoter CpG island methylation markers in colorectal cancer: the road ahead. Epigenomics 4(2), 179-194 (2012).

3 Hitchins MP, Rapkins RW, Kwok CT et al. Dominantly inherited constitutional epigenetic silencing of MLH1 in a cancer-affected family is linked to a single nucleotide variant within the 5'UTR. Cancer Cell 20(2), 200-213 (2011).

4 Mrkonjic M, Roslin NM, Greenwood CM et al. Specific variants in the $M L H 1$ gene region may drive DNA methylation, loss of protein expression, and MSI-H colorectal cancer. PLoS ONE 5(10), e13314 (2010).

5 Cui H, Cruz-Correa M, Giardiello FM et al. Loss of IGF2 imprinting: a potential marker of colorectal cancer risk. Science 299 , 1753-1755 (2003).

6 Febbo PG, Ladanyi M, Aldape KD et al. NCCN Task Force report: evaluating the clinical utility of tumor markers in oncology. J. Natl Compr. Canc. Netw. 9(Suppl. 5), S1-32; quiz S33 (2011).

7 Schwarzenbach H, Hoon DS, Pantel K. Cellfree nucleic acids as biomarkers in cancer patients. Nat. Rev. Cancer 11(6), 426-437 (2011).

8 Herbst A, Wallner M, Rahmig K et al. Methylation of helicase-like transcription factor in serum of patients with colorectal cancer is an independent predictor of disease recurrence. Eur. J. Gastroenterol. Hepatol. 21(5), 565-569 (2009).

9 Lofton-Day C, Model F, Devos T et al. DNA methylation biomarkers for blood-based colorectal cancer screening. Clin. Chem. 54(2), 414-423 (2008).

10 Ahlquist DA, Zou H, Domanico M et al. Next-generation stool DNA test accurately detects colorectal cancer and large adenomas. Gastroenterology 142(2), 248-256 (2012).

11 Warren JD, Xiong W, Bunker AM et al. Septin 9 methylated DNA is a sensitive and specific blood test for colorectal cancer. $B M C$ Med. 9, 133 (2011).
12 Bosch LJ, Oort FA, Neerincx M et al. DNA Methylation of phosphatase and actin regulator 3 detects colorectal cancer in stool and complements FIT. Cancer Prev. Res. (Phila) 5(3), 464-472 (2012).

13 Ahlquist DA, Taylor WR, Mahoney DW et al. The stool DNA test is more accurate than the plasma septin 9 test in detecting colorectal neoplasia. Clin. Gastroenterol. Hepatol. 10(3), 272.e1-277.e1 (2012).

14 Lofton-Day C, Model F, Devos T et al. DNA methylation biomarkers for bloodbased colorectal cancer screening. Clin. Chem. 54(2), 414-423 (2008).

15 Tang D, Liu J, Wang DR, Yu HF, Li YK, Zhang JQ. Diagnostic and prognostic value of the methylation status of secreted frizzled-related protein 2 in colorectal cancer. Clin. Invest. Med. 34(2), E88-E95 (2011).

16 Lange CP, Campan M, Hinoue T et al. Genome-scale discovery of DNAmethylation biomarkers for blood-based detection of colorectal cancer. PLoS ONE 7(11), e50266 (2012). 
17 Toyota M, Ahuja N, Ohe-Toyota M, Herman JG, Baylin SB, Issa JP. CpG island methylator phenotype in colorectal cancer. Proc. Natl Acad. Sci. USA 96, 8681-8686 (1999).

18 Weisenberger DJ, Siegmund KD, Campan M et al. $\mathrm{CpG}$ island methylator phenotype underlies sporadic microsatellite instability and is tightly associated with BRAF mutation in colorectal cancer. Nat. Genet. 38, 787-793 (2006).
19 Issa JP. CpG island methylator phenotype in cancer. Nat. Rev. Cancer 4, 988-993 (2004).

20 Ribic CM, Sargent DJ, Moore MJ et al. Tumor microsatellite-instability status as a predictor of benefit from fluorouracil-based adjuvant chemotherapy for colon cancer. N. Engl. J. Med. 349(3), 247-257 (2003).

21 Van Rijnsoever M, Elsaleh H, Joseph D, Mccaul K, Iacopetta B. CpG island methylator phenotype is an independent predictor of survival benefit from 5 -fluorouracil in stage III colorectal cancer. Clin. Cancer Res. 9(8), 2898-2903 (2003).

22 Jover R, Nguyen TP, Perez-Carbonell L et al. 5-Fluorouracil adjuvant chemotherapy does not increase survival in patients with $\mathrm{CpG}$ island methylator phenotype colorectal cancer. Gastroenterology 140(4), 1174-1181 (2011).

23 Ebert MP, Tanzer M, Balluff B et al. TFAP2E-DKK4 and chemoresistance in colorectal cancer. N. Engl. J. Med. 366(1), 44-53 (2012). 